\title{
Using decision analysis to improve malaria control policy making
}

\author{
Randall A. Kramer ${ }^{\mathrm{a}, *}$, Katherine L. Dickinson ${ }^{\mathrm{b}}$, Richard M. Anderson ${ }^{\mathrm{c}}$, Vance G. Fowler ${ }^{\mathrm{d}}$, \\ Marie Lynn Miranda ${ }^{c}$, Clifford M. Mutero ${ }^{\mathrm{e}}$, Kathryn A. Saterson ${ }^{\mathrm{f}}$, Jonathan B. Wiener ${ }^{\mathrm{g}}$ \\ a Nicholas School of the Environment, Box 90328, Duke University, Durham, NC 27708, USA \\ b Department of Population Health Sciences, University of Wisconsin, Madison, WI, USA \\ c Nicholas School of the Environment, Duke University, Durham, NC, USA \\ d Department of Medicine, Division of Infectious Diseases, Duke University Medical Center, Durham, NC, USA \\ e Africa Policy Institute, Pretoria, South Africa \\ f National Health and Environmental Effects Research Laboratory, United States Environmental Protection Agency, Research Triangle Park, NC, USA \\ g Duke University Law School, Nicholas School of the Environment, and Sanford Institute of Public Policy, Duke University, Durham, NC, USA
}

\section{A R T I C L E I N F O}

\section{Keywords:}

Malaria

Decision analysis

Health policy

Cost-effectiveness

Environmental impacts

Benefit-cost analysis

\begin{abstract}
A B S T R A C T
Malaria and other vector-borne diseases represent a significant and growing burden in many tropical countries. Successfully addressing these threats will require policies that expand access to and use of existing control methods, such as insecticide-treated bed nets (ITNs) and artemesinin combination therapies (ACTs) for malaria, while weighing the costs and benefits of alternative approaches over time. This paper argues that decision analysis provides a valuable framework for formulating such policies and combating the emergence and re-emergence of malaria and other diseases. We outline five challenges that policy makers and practitioners face in the struggle against malaria, and demonstrate how decision analysis can help to address and overcome these challenges. A prototype decision analysis framework for malaria control in Tanzania is presented, highlighting the key components that a decision support tool should include. Developing and applying such a framework can promote stronger and more effective linkages between research and policy, ultimately helping to reduce the burden of malaria and other vector-borne diseases.
\end{abstract}

(c) 2009 Elsevier Ireland Ltd. All rights reserved.
Key messages:

1. Effective control of malaria and other vector-borne diseases calls for policy making that considers the costs and benefits of various control methods.

2. Decision analysis provides a valuable framework for formulating such policies and combating the emergence and re-emergence of malaria and other diseases.

3. Developing and applying a decision analysis framework can promote stronger and more effective linkages between research and policy, ultimately helping to reduce the burden of malaria and other vector-borne diseases.

\footnotetext{
* Corresponding author. Tel.: +1 919613 8072; fax: +1 9196810361. E-mail address: kramer@duke.edu (R.A. Kramer).
}

\section{Introduction}

Vector-borne diseases affect millions of people every year, mostly in tropical countries. In particular, malaria is the most deadly vector-borne disease, killing over one million people annually [1]. After substantial progress in battling the spread of malaria in the 1960s and 1970s, the number of reported malaria cases and the geographic extent of the disease grew dramatically in the past 25 years. Beyond mortality losses, malaria imposes devastating costs on local economies, through direct costs of treatment and prevention, indirect costs of lost productivity, and lower economic growth at the national and regional level [2,3].

In the face of this substantial and growing public health challenge, many have emphasized the need for new and improved "technological" solutions such as malaria vaccines and genetic modification of vector 
populations [4,5]. However, it is important to recognize that the malaria threat has continued to grow despite the existence of effective control technologies such as insecticide-treated bed nets (ITNs), indoor residual spraying (IRS), artemesinin combination therapies (ACTs), and rapid diagnostic tests. While there is evidence that each of these approaches may be effective in combating malaria, significant implementation challenges have prevented more widespread adoption of potentially effective solutions. Without more attention to these policy challenges, malaria control efforts will be severely hampered.

This paper examines the application of decision analysis methods to address the challenges of developing and implementing more effective malaria control policies. We enumerate key challenges that policy makers and practitioners face in their efforts to combat malaria and other vector-borne diseases. We argue that a comprehensive decision analysis framework can help to address these challenges, guiding the selection of more effective, evidence-based control strategies. After developing theoretical and practical arguments for the decision analysis approach, we present a prototype malaria decision analysis support tool (MDAST) for Tanzania.

\section{A need for decision analysis to guide control of malaria and other vector-borne diseases}

Malaria poses a unique set of challenges that limit decision makers' ability to effectively confront and control the burden of this disease. Decision analysis offers several advantages that are particularly suited to the complex problem of controlling malaria and other vector-borne diseases [6]. Decision analysis is a structured approach to making choices, enabling a systematic evaluation of the consequences of alternative courses of action that one might take in the face of uncertainties about outcomes [7]. Table 1 summarizes five critical challenges that characterize vector-borne disease control policy, and explains how a decision analysis framework could address these challenges.

\subsection{High stakes environment}

Vector-borne diseases place a significant and growing burden on human populations across the globe. Given the severity and extent of these problems, developing sound strategies for controlling vector-borne diseases is of the utmost importance. Ineffective, uninformed policies can have devastating consequences. Resource-poor countries, in particular, simply cannot afford to make uninformed choices in combating these diseases. The stakes for successfully combating this disease are particularly high for children under five; in Tanzania, for example, $45 \%$ of all malaria cases occur in this age group [8]. By providing a framework for carefully analyzing the alternatives and examining their effects on a range of outcomes over time, decision analysis provides a basis for informed decision making in this high stakes environment.

\subsection{Multiple actors at multiple scales}

Combating vector-borne diseases involves multiple actors at multiple scales, including international donor organizations, national governments, universities, nongovernmental organizations, local health providers, and individual communities and households. These actors vary in terms of their perspectives and priorities, creating a difficult task for decision makers charged with selecting a particular control strategy. In particular, decision makers may feel significant pressure from different stakeholders to adopt one approach or another. International donors may favor an approach that conflicts with the preferences of national agencies or local communities. Decision makers must also select the best strategy for a particular area, with the "best" strategy highly dependent on local conditions.

By providing decision makers with a framework for using evidence on the effectiveness and cost of different interventions, decision analysis facilitates more informed policy making. In addition, decision analysis structures the decision problem, clarifying the relative importance of contributing factors within the local context. In this way, decision analysis provides a unifying tool that brings multiple actors together to productively address vector-borne diseases.

Table 1

Summary of arguments for applying decision analysis to vector-borne disease control.

\begin{tabular}{ll}
\hline Challenges in combating vector-borne diseases & What decision analysis offers \\
\hline $\begin{array}{l}\text { 1. The growing burden of malaria and other vector-borne } \\
\text { diseases creates a high-stakes environment where bad } \\
\text { policy decisions are extremely costly }\end{array}$ & $\begin{array}{l}\text { By promoting informed, evidence-based policies, decision analysis can } \\
\text { improve the allocation of limited resources for reducing the burden of } \\
\text { malaria and other vector-borne diseases } \\
\text { actors at multiple scales }\end{array}$ \\
$\begin{array}{l}\text { Decision analysis can provide a focal point for discussions among } \\
\text { making difficult tradeoffs among competing health, social, } \\
\text { and environmental objectives }\end{array}$ & $\begin{array}{l}\text { Decision analysis directly identifies competing objectives and helps } \\
\text { decision makers to confront tradeoffs }\end{array}$ \\
$\begin{array}{l}\text { 4. Complicated dynamics, interdependencies, and } \\
\text { uncertainties make it difficult to analyze the effects of } \\
\text { vector-borne disease control strategies over time }\end{array}$ & $\begin{array}{l}\text { Sophisticated decision analysis models incorporate multiple layers of } \\
\text { detail to reflect the complexity of the vector-borne disease control } \\
\text { problem. Simulations and sensitivity analyses allow decision makers to } \\
\text { explore possible effects of different strategies over time } \\
\text { Decision analysis frameworks can be structured to bring together } \\
\text { human-environment interactions that necessitate } \\
\text { interagency, interdisciplinary analyses and responses }\end{array}$ \\
& $\begin{array}{l}\text { multiple perspectives and areas of expertise, thus fostering } \\
\text { collaboration and dialogue to accurately represent and address } \\
\text { vector-borne disease control }\end{array}$ \\
\hline
\end{tabular}




\subsection{Complex tradeoffs}

Addressing vector-borne diseases often requires tradeoffs among competing fundamental objectives. This is particularly evident, for example, in the debate over the use of the pesticide DDT to combat malaria [9-11]. The malaria-DDT dilemma presents a classic risk-risk tradeoff $[12,13]$. In this case, the immediate human health risk posed by malaria must be weighed against possible longer term risks to human health and ecosystems. In East Africa, the debate over the use of DDT for malaria control continues. Under the Stockholm Convention on Persistent Organic Pollutants (POPs), countries are authorized to elect further use of DDT for malaria vector control when locally safe, effective, and affordable alternatives are not available. Uganda has decided to allow the reintroduction of DDT for malaria control, and some reports indicate that the public reaction has been strongly positive [14]. In Tanzania, policy makers are considering reintroducing DDT for indoor residual spraying in epidemic areas [6]. Meanwhile, most Kenyan policy makers are still firmly opposed to DDT use [15].

Decision analysis tools can inform debates by separating issues of scientific uncertainty (e.g., what is the specific impact of DDT on human and ecosystem health when used in a particular way?) from disagreement over values (e.g., how much weight should we put on human health vs. environmental impacts?). While decision analysis on its own cannot resolve these disagreements, highlighting the role of these different components and providing a user-friendly tool that allows people to explore the impact of different component weights on the optimal policy choice, creates a more systematic mechanism for analyzing alternatives and making informed tradeoffs.

\subsection{Dynamics, interdependencies, and uncertainties}

Vector-borne diseases are characterized by complicated dynamics that make it difficult to predict the future consequences of current policy decisions. In particular, strategies that are effective today may become less so in the future as resistance to drugs and insecticides develops [16]. Given the overall prevalence of malaria in this region, resistance to antimalarial drugs has been particularly problematic in East Africa. For example, chloroquine resistance has spread much more rapidly in East Africa than West Africa, prompting countries like Uganda and Kenya to change their antimalarial drug policies [17]. These resistance problems create dynamic challenges for policy makers in determining the optimal set of malaria policies over time. Dynamic decision analysis models can help decision makers explore the possible impacts of alternative current choices over different time horizons. Policy simulations can formally introduce scientific uncertainty and thus provide information on the bounds on the problem.

\subsection{Complex human-environment interactions}

As with many other problems, current attempts to combat vector-borne diseases often address the problem from the perspective of single disciplines or individual government agencies, none of which are fully equipped to understand and address these diseases in their entirety. To highlight one problem, current strategies for controlling vector-borne diseases tend to focus on either (1) controlling the disease vector, or (2) preventing and treating the disease itself. One group of people, (e.g., entomologists and environmental managers) focus on the former strategy, while the medical community tends to focus on the latter. A third important component of vector-borne disease control, understanding social factors and behavior change, receives little systematic attention from either group [18]. Decision makers are left with few tools for comparing alternative strategies and understanding how, for example, vector control efforts might interact with disease management in a particular social and cultural setting.

Complex human-environment interactions constitute a fundamental component of the malaria problem in East Africa. In particular, complicated relationships exist between land use change for agriculture and malaria outcomes. In the highlands of Uganda, for example, converting natural swamps to agriculture led to an increase in temperatures and mosquito vectors, leading to higher malaria risks in these areas compared to areas where natural swampland was maintained [19]. Larger scale phenomena are also likely to play a key role in changing the relationships among humans, the environment, and malaria over time. For example, studies have found that climate change may play a significant role in the increase of malaria epidemics in the East African Highlands [20,21]. A decision analysis approach to malaria control should capture the interactions between the disease and several environmental variables (e.g., global or local climate change, deforestation, and irrigation) [22,23].

Decision analysis tools are uniquely suited to address all five of the vector-borne disease control challenges outlined here. By providing a systematic means of combining an evolving knowledge base from research in many different fields, decision analysis can encourage greater use of evidence-based decision making in the complex, high stakes environment that characterizes malaria policy making. Furthermore, such tools provide a framework for promoting dialogue across the multiple stakeholders at various levels of policy making. A decision analysis approach is also consistent with the call by WHO for the implementation of a Global Strategic Framework for Integrated Vector Management and with efforts of the Innovative Vector Control Consortium and the Malaria Transmission Consortium to address the challenges of controlling of vector-borne diseases through evidence-based decision making [24].

\section{Demand for decision analysis tools among East African stakeholders}

To gauge the degree of interest in a decision support approach to malaria control, preliminary field work was conducted in Tanzania, Uganda, and Kenya in 2005-2007. Our interdisciplinary research team queried policy makers, researchers, and other stakeholders with semi-structured interviews and a small Internet survey of a dozen experts. We also conducted a workshop in Dar es Salaam in June 2006, and presented our conceptual framework to a national medical research conference in Arusha, Tanzania 


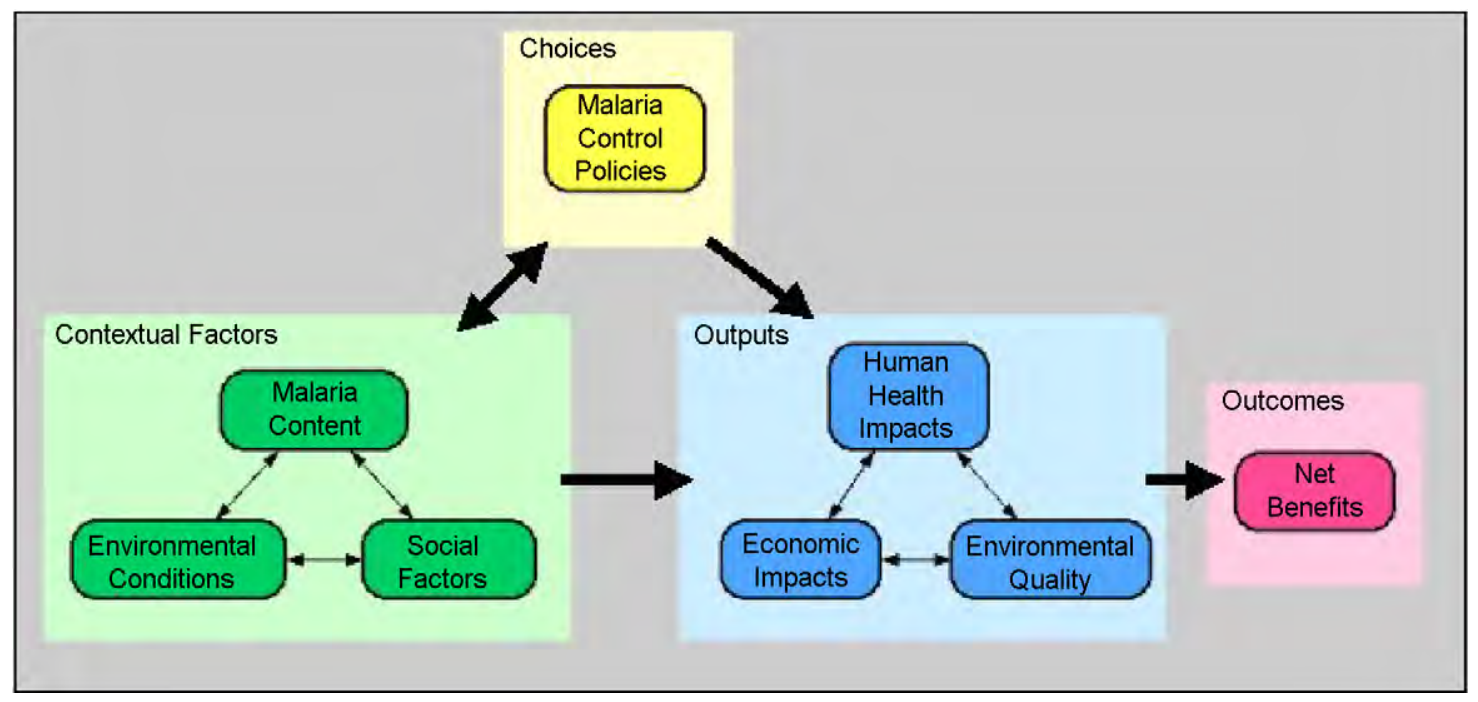

Fig. 1. Overview of prototype MDAST model showing relationships among: malaria control policies; contextual factors and intermediate processes; health, environmental, and social outputs; and final outcomes.

in March 2007. In total, more than 50 key informants provided feedback to the research team through workshops, interviews, and the survey.

Overall, informants expressed a high level of support for using decision analysis to inform malaria control policymaking. Informants repeatedly stressed the need for ways to connect research and policy to inform malaria control decisions. In our 2007 Internet survey of Tanzanian researchers and policy makers, most respondents felt that donor preferences currently play the largest role in determining malaria control policies, but most felt that scientific findings should play the largest role. At the 2007 national medical conference in Arusha, Tanzania, the final session included considerable discussion about the potential role that that decision analysis could play in improving malaria policy making. Thus, overall support for a decision analysis approach to informing malaria control policy making was quite high.

East African researchers and policy makers also stressed the importance of each of the key challenges listed in the previous section. In numerous interviews, informants called attention to the severity and urgency of the malaria problem in their countries noting the human health, social development and economic performance impacts. Other informants stressed that given the recent, rapid expansion in donor support for malaria control, there is a need to move quickly to provide policy relevant information in a timely fashion. Informants emphasized that the increase in funding and research results is creating both a dynamic policy environment and a strong need for comprehensive tools to better inform policy making through systematic assessment of the array of new choices.

East African policy makers and researchers also called attention to the number of actors that are involved in malaria control policy making, and the difficulty of incorporating and responding to their sometimes conflicting needs. There was a prevailing view that current decision making on malaria control is fragmented, often focusing on one intervention at a time, and responding to the latest donor emphasis and pressure.

According to the experts who responded to the Internet survey, the top three objectives policy makers currently consider when deciding among malaria control policies are reducing malaria prevalence/incidence, minimizing costs, and reducing the risk of epidemics.

\section{The malaria decision analysis support tool (MDAST): a prototype}

In this section, we describe a prototype malaria decision analysis support tool that has been developed with the assistance of malaria decision makers in Tanzania. The development of MDAST draws on other applications of decision analysis to health issues in developing countries. For example, Hu et al. used decision analysis to study the policy implications of HIV infection and breastfeeding [25]. Bertolli et al. developed a decision analysis approach to examine a range of interventions to reduce mother-to-child HIV transmission [26]. Unlike these studies, which sought to minimize a single health outcome (childhood mortality), our malaria decision support tool incorporates multiple objectives including human health outcomes, ecological risks, and economic costs. MDAST is also designed to examine "risk-risk tradeoffs" [13]. For example, spraying DDT may reduce malaria burden but increase risks to wildlife; ceasing the use of DDT may reduce risks to wildlife but increase malaria burden. Or, draining wetlands may reduce the spread of mosquitoes but harm other biota. Whereas government policy often proceeds by identifying a target risk and neglecting ancillary or countervailing factors, risk-risk analysis counsels a more holistic approach to the multiple consequences of decisions in complex systems [13,27], MDAST helps decision makers identify these diverse effects, weigh the tradeoffs, and, in the longer run, seek "risk-superior moves" that reduce multiple risks in concert [12]. Fig. 1 provides an overview of 


\section{Tanzania: Distribution of Endemic Malaria}

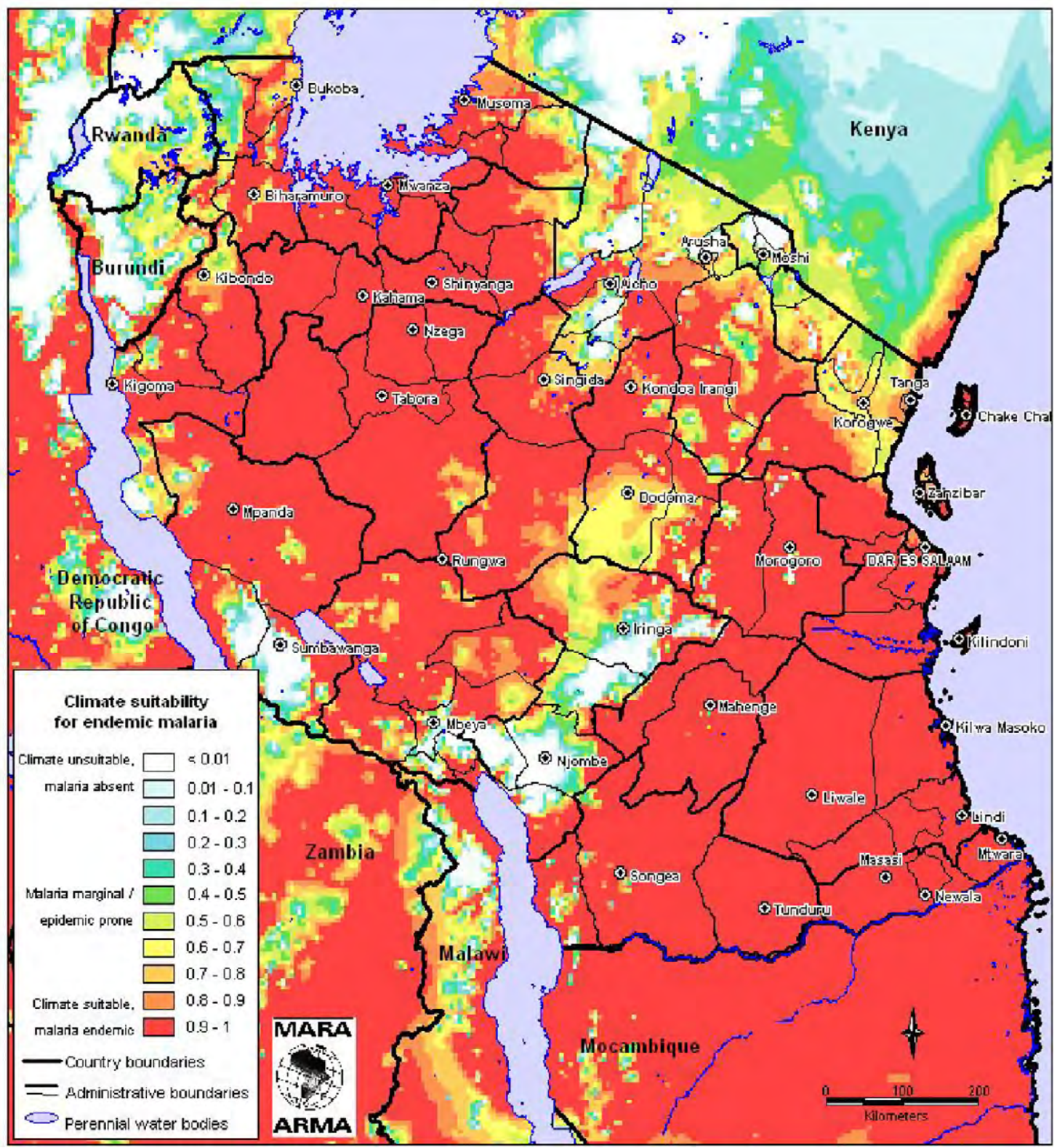

Fig. 2. Climate suitability for endemic malaria across Tanzania. Source: MARA/ARMA 2002.

the prototype MDAST. This overview figure highlights some of the key components that are included in the decision tool.

\subsection{Choices: malaria control policies}

While decision makers currently make use of whatever information they have available on the effectiveness of different interventions, the purpose of MDAST is to allow a more systematic exploration of the likely impacts of different malaria control interventions on outcomes of interest. The decision tool includes a range of malaria control options that decision makers have at their disposal, including both disease management and vector control methods. MDAST addresses a broad range of policies that combine technological tools as well as delivery mechanisms and incentives. For vector control, key technologies that are analyzed include insecticide-treated nets and indoor residual spraying, as well as environmental management (e.g., larviciding, draining standing areas of water, and changing irrigation practices). Over longer time horizons, strategies like genetic modification of mosquito populations may come into play. For disease prevention and treatment, MDAST examines artemesinin combination therapy (ACT), intermittent preventative treatment for pregnant women, and use of rapid diagnostic tests. As vaccines become available, they can 


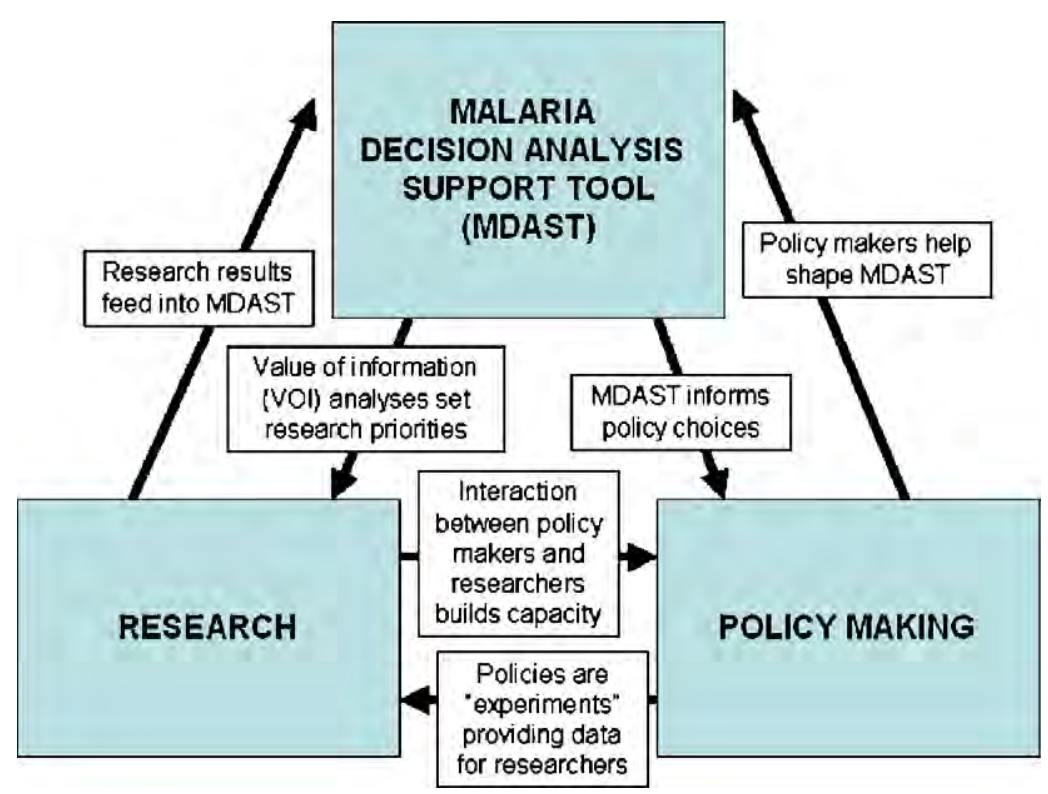

Fig. 3. MDAST promotes dynamic linkages between research and policy.

be added as another choice option. For each of these technologies, MDAST allows analysis of different ways to implement the technology and deliver it to the target population. For example, will ITNs be given away for free or at reduced cost to pregnant women and children under five? Will they be distributed through the public or the private sector?

\subsection{Contextual factors: malaria context}

The effects and effectiveness of malaria control policies depends on several contextual factors. The malaria context encompasses transmission intensity and severity as well as the number and species composition of malaria vectors (mosquitoes). As Fig. 2 illustrates, several aspects of the malaria context may vary over space. In Tanzania, malaria is endemic throughout most of the country, but there are also several areas where transmission is less stable and epidemics are the main concern. The prototype MDAST takes this spatial heterogeneity into account by allowing the user to input localized parameters for a particular region or district.

\subsection{Contextual factors: environmental conditions}

Closely related to the malaria context, the nature and characteristics of the climate and landscape in different regions create different "eco-epidemiological settings" [28] with resulting variation in malaria transmission and treatment options. Fig. 2 shows how variation in ecological zones and climate conditions in Tanzania produce variation in the distribution of endemic malaria. Other environmental factors, such as the type of agriculture practiced, also affect both conditions for malaria transmission and opportunities for malaria control. For example, some modern rice farming systems in Mvomero District in Tanzania are experimenting with the use of a nitrogen-fixing plant called azolla to simultaneously fertilize rice paddies and prevent mosquito larvae growth [29]. When more fully developed, MDAST will incorporate these environmental factors in analyzing the potential impact of different malaria control policies in different areas.

\subsection{Contextual factors: social factors}

Local behavior and social factors are often overlooked by policy makers in designing malaria control strategies [18]. These factors play a critical role in determining how health policies (e.g., free distribution of ITNs to all pregnant women and children under five) translate into practice on the ground (e.g., number of pregnant women and children under five sleeping beneath ITNs on a regular basis). For the case of ITNs, a large-scale trial carried out in western Kenya showed that even when nets were given away for free, approximately $30 \%$ of ITNs were unused, and factors such as temperature, individuals' ages, and sleeping arrangements had a significant impact on use rates [30]. An analysis of the potential effects of malaria control policies that ignores these behavioral and social factors will produce flawed policy recommendations. Thus, MDAST must reflect the key role that local culture, knowledge, and beliefs play in determining how individuals and households perceive and respond to the malaria threat.

\subsection{Outputs: human health impacts}

The purpose of MDAST is to analyze the likely impacts of different malaria control policies on different outputs of interest. The malaria burden in the human population is clearly one of the impacts policy makers are most concerned with in deciding among various malaria control strategies. This burden is a function of the malaria con- 
text and the resulting infection rate, in addition to disease treatment strategies. By modeling the linkages between contextual factors and the malaria burden, and allowing decision makers to examine "portfolios" of malaria control options, MDAST enables analyses of dynamic effects and interactions between vector control and disease management. For example, prompt and effective diagnosis and treatment of malaria cases will decrease the malaria burden in the current period, and may also prevent future cases by reducing the overall parasite load in the population. Furthermore, large investments in vector control will result in fewer malaria cases, possibly decreasing the demand for treatment and freeing up health care system resources. Allowing policy makers to examine these feedbacks and interactive effects is one of the key advantages of MDAST approach.

\subsection{Outputs: environmental quality}

While the primary focus of malaria control policies is generally and appropriately on reducing the malaria burden, there is increasing awareness of the potential for adverse environmental impacts from different approaches to controlling vectors, which can then have additional health and cost results. For example, if insecticides used for IRS escape into the environment through improper cleaning and disposal of insecticide canisters or through improper use in agriculture, water quality may decline.

\subsection{Outputs: economic impacts}

Policy makers everywhere face limited budgets, and this is particularly true in East Africa. Calculating the economic impacts of different malaria control strategies is clearly important, and the prototype MDAST allows policy makers to estimate and compare the amount of financial resources that are needed to maintain different combinations of control techniques over time. The prototype version of MDAST takes a broad view of costs, including the costs of materials and supplies (e.g., nets, drugs) as well as human resources and other costs (e.g., training people to conduct IRS, net retreatment, compliance improvement programs) [31]. Avoided mortality is evaluated in monetary terms using literature estimates of the value of a statistical life (VSL) while avoided mortality is estimated by applying available cost of illness (COI) parameters to the number of avoided symptomatic cases [32-35]. Future model work will focus on the economic burden of the disease in the form of reduced agricultural productivity, lost schooling, and lost wages. We will also develop a module to calculate the total environmental impact cost to allow decision makers to consider how malaria interventions affect environmental indicators like water quality. Finally, if DDT use leads to decreased agricultural trade, this would be an additional economic impact that should be modeled.

\subsection{Outcomes: net benefits}

MDAST allows decision makers to examine the potential impacts of different malaria control strategies on several key outputs of interest, including health outcomes as well as social, environmental, and economic impacts. For key variables, outcomes are presented in the form of probability distributions rather than deterministically, thereby capturing the impact of various sources of uncertainty in the process linking policies to outcomes. Comparing these probability distributions over the impacts of different sets of policies allows policy makers to examine tradeoffs among different objectives. For example, MDAST may suggest that Policy A (e.g., large investment in IRS, in combination with ACTs) will result in reductions in malaria mortality, but entail high costs and a significant risk to environmental quality, while Policy B (e.g., environmental management, ITNs, and continued reliance on sulphadoxine-pyrimethamine (SP) drug treatment) requires fewer resources and poses a smaller environmental threat but results in more variable health impacts. Physical impacts (such as mortality) are identified and reported as well as the economic valuations of those impacts, so that users of MDAST can make choices about the appropriate valuation methods to employ in their settings.

\section{Conclusions: using MDAST to promote evidence-based policy and policy-relevant research}

Moving from the prototype to a more elaborated MDAST for Tanzania, we are focusing on making the tool more empirically based. We are currently engaged in:(1) expanding the prototype MDAST model components to better capture the specific processes involved in malaria transmission and control, and (2) populating the model using a number of datasets, including environmental variables, behavioral and social factors, data on malaria vectors and malaria cases, and costs and poverty data. In some cases, expert judgment and elicitation are being used to provide model inputs for which data are not available. The completed MDAST model will be a flexible and versatile tool that can be applied at different scales (e.g., national, regional, local) and take inputs from various locations to meet decision makers'.

The immediate purpose of MDAST is to provide policy makers with a sound method for translating a vast set of research results and expert opinion into evidencebased policy decisions. In addition to fulfilling this need, MDAST can serve a broader role in forging links between research and policy (see Fig. 3). First, as already described, MDAST can play an important role in informing policy decisions by providing information on the probable outcomes of different combinations of malaria control strategies. In turn, MDAST relies on input from policy makers and practitioners to ensure that we are striking the right balance between complexity/accuracy and tractability/usability. Second, research results, including data sets and results of expert elicitations, provide the empirical basis for MDAST's policy recommendations. In turn, MDAST can provide useful information on priority research areas by serving as the basis for future value of information (VOI) analyses to help identify the variables for which the acquisition of additional information would most improve policy decisions. Finally, by bringing research and policy closer together MDAST can highlight and foster other key interactions. Allowing policy makers to work more closely with researchers and to better 
understand the implications of research results for policy decisions serves a key capacity-building role on both sides. Furthermore, research can take advantage of the opportunity that policy "experiments" present for gathering data and analyzing the impacts of different interventions.

Malaria and other vector-borne diseases present a pressing challenge for policy makers and practitioners tasked with combating and eliminating these illnesses. In this complex, high-stakes environment, tools that help decision makers compare alternative policy options and make better choices are essential. We believe that the application of decision analysis methods to the complex problem of malaria control presents a promising opportunity to improve health policy decision making.

\section{Acknowledgements}

This research was supported by funding from the National Institute of Environmental Health Sciences, 1 P30 ES-011961-01A1, and from the Duke Provost Common Fund.

\section{References}

[1] Gubler DJ. Resurgent vector-borne diseases as a global health problem. Emerging Infectious Diseases 1998;4(3):442-50.

[2] Gallup JL, Sachs JD. The economic burden of malaria. American Journal of Tropical Medicine and Hygiene 2001;64(1-2):85-96.

[3] WHO. Roll back malaria program fact sheet \#94 "malaria in Africa"; 2003. Cited; available from: http://www.rbm.who.int/cmc upload/0/000/015/370/RBMInfosheet_3.htm.

[4] Matuschewski K, Mueller AK. Vaccines against malaria - an update. FEBS Journal 2007;274(18):4680-7.

[5] Catteruccia F. Malaria vector control in the third millennium: progress and perspectives of molecular approaches. Pest Management Science 2007;63(7):634-40.

[6] Mboera L. Decision analysis in malaria vector control programmes. Tanzania Health Research Bulletin 1998;1(2):18-26.

[7] Keeney RL, Hammond JS, Raiffa H. Smart choices: a practical guide to making better decisions. Harvard Business School Press; 1999.

[8] WHO, World malaria report 2005 - United Republic of Tanzania Country Profile; 2005.

[9] Raloff J. The case for DDT. Science News 2000;158(1):12.

[10] Liroff RA. DDT risk assessments: response. Environmental Health Perspectives 2001;109(7):A302-a303.

[11] Tren R, Bate R. Malaria and the DDT story. London: Institute of Economic Affairs; 2001.

[12] Graham JD, Wiener JB. Risk vs. risk: tradeoffs in protecting health and the environment. Harvard University Press; 1995.

[13] Wiener JB. Managing the iatrogenic risks of risk management. Risk: Health Safety \& Environment 1998;9:39-84.

[14] Ssenkabirwa A-M, Kibuuka S. Ugandans applaud DDT clearance. In: The monitor; Kampala; 2006.

[15] Biscoe ML, Mutero CM, Kramer RA. Current policy and status of DDT use for malaria control in Ethiopia, Uganda, Kenya and South Africa. Colombo, Sri Lanka: International Water Management Institute; 2005.

[16] Fowler VG, Lemnge M, Irare SG, Malecela E, Mhina J, Mtui S, et al. Efficacy of chloroquine on plasmodium-falciparum transmitted at Amani, Eastern Usambara Mountains, Northeast Tanzania - an area where malaria has recently become endemic. Journal of Tropical Medicine and Hygiene 1993;96(6):337-45.
[17] D'Alessandro U, Buttiens H. History and importance of antimalarial drug resistance. Tropical Medicine \& International Health 2001;6(11):845-8.

[18] Heggenhougen HK, Hackethal V, Vivek P. The behavioural and social aspects of malaria and its control. UNDP/World Bank/WHO: Special Programme for Research and Training in Tropical Diseases (TDR); 2003.

[19] Lindblade KA, Walker ED, Onapa AW, Katungu J, Wilson ML. Land use change alters malaria transmission parameters by modifying temperature in a highland area of Uganda. Tropical Medicine \& International Health 2000;5(4):263-74.

[20] Koenraadt CJM, Paaijmans KP, Schneider P, Githeko AK, Takken W. Low larval vector survival explains unstable malaria in the western Kenya highlands. Tropical Medicine \& International Health 2006;11(8):1195-205.

[21] Zhou G, Minakawa N, Githeko AK, Yan G. Association between climate variability and malaria epidemics in the East African highlands. Proceedings of the National Academy of Sciences USA 2004;101(8):2375-80.

[22] Pattanayak S, Dickinson K, Corey C, Murray B, Sills E, Kramer RA. Deforestation, malaria, and poverty: a call for transdisciplinary research to support the design of cross-sectoral policies. Sustainability: Science, Practice, and Policy 2006;2(2):45-56.

[23] Patz JA, Daszak P, Tabor GM, Alonso Aguirre A, Pearl M, Epstein J, et al. Unhealthy landscapes: policy recommendations on land use change and infectious disease emergence. Environmental Health Perspectives 2004;112(10):1092-8.

[24] WHO. Global strategic framework for integrated vector management. Geneva: World Health Organization; 2004.

[25] Hu DJ, Heyward WL, Byers RH, Nkowane BM, Oxtoby MJ, Holck SE, et al. HIV-infection and breast-feeding - policy implications through a decision-analysis model. AIDS 1992;6(12):1505-13.

[26] Bertolli J, Hu DJ, Nieburg P, Macalalad A, Simonds RJ. Decision analysis to guide choice of interventions to reduce mother-to-child transmission of HIV. AIDS 2003;17(14):2089-98.

[27] Wiener JB. Precaution in a multi-risk world. In: Paustenbach DD, editor. Human and ecological risk assessment: theory and practice. New York: John Wiley \& Sons; 2002. p. 1509-31.

[28] Keiser J, Singer BH, Utzinger J. Reducing the burden of malaria in different eco-epidemiological settings with environmental management: a systematic review. Lancet Infectious Diseases 2005;5(11): 695-708.

[29] Mlozi MRS, Shayo EH, Senkoro KP, Mayala BK, Rumisha SF, Mutayoba B, et al. Participatory involvement of farming communities and public sectors in determining malaria control strategies in Mvomero District Tanzania. Tanzania Health Research Bulletin 2006;8(3): 134-40.

[30] Alaii JA, Hawley WA, Kolczak MS, ter Kuile FO, Gimnig JE, Vulule JM, et al. Factors affecting use of permethrin-treated bed nets during a randomized controlled trial in western Kenya. American Journal of Tropical Medicine and Hygiene 2003;68(4):137-41.

[31] Goodman C, Coleman PG, Mills A. Economic analysis of malaria control in sub-Saharan Africa. Global Forum for Health Research 2000, http://rbm.who.int/cmc_upload/0/000/015/601/ec_analysis_ssa.pdf.

[32] Markandya A. The valuation of health impacts in developing countries. Planejamento e Politicas Publicas 1998;18:119-58.

[33] Asante F, Asenso-Okyere K. The economic burden of malaria in Ghana. Lagon: Institute of Statistical, Social and Economic Research (ISSER), University of Ghana; 2003.

[34] Deressa W, Hailemariam D, Ali A. Economic costs of epidemic malaria to households in rural Ethiopia. Tropical Medicine \& International Health 2007; 12(10):1148-56.

[35] Kochi I, Hubbel B, Kramer R. An empirical bayes approach to combining and comparing estimates of the value of a statistical life for environmental policy analysis. Environmental and Resource Economics 2006;34:385-406. 